\section{Deterioro neurocognoscitivo por exposición al plomo en hijos de alfareros andinos}

Todavía no se ha determinado con certeza la concentración de plomo en el organismo que deteriora la capacidad cognoscitiva. Según algunos estudios con pruebas de inteligencia basadas en la comprensión del habla, ese deterioro puede asociarse con concentraciones pediátricas de plomo en la sangre menores de $10 \mu \mathrm{g} / \mathrm{dL}$. Sin embargo, es posible que en esas pruebas se produzcan sesgos culturales al evaluar el funcionamiento mental. Por esta razón se realizó un nuevo estudio de los efectos de la concentración de plomo en el cociente intelectual (CI) de niños andinos que habían sufrido una exposición ambiental crónica a ese metal, pero esta vez la prueba del funcionamiento cognoscitivo no dependía del lenguaje. Se usó la prueba de matrices progresivas de Raven en color (Raven's Colored Progressive Matrices o RCPM), la cual no es verbal.

Participaron 188 niños de 5,33 a 11,67 años, todos con exposición crónica al plomo, pues eran hijos de ecuatorianos andinos que trabajaban en la industria de la alfarería vidriada. Los resultados indicaron que la concentración sanguínea media de plomo de los niños era 29,3 $\mu \mathrm{g} / \mathrm{dL}$ (recorrido de 3,5 a 94,3). Cuarenta y siete niños tenían concentraciones de plomo menores de $10 \mu \mathrm{g} / \mathrm{dL}$ (clasificación 1 de los Centros para el Control y la Prevención de Enfermedades de los Estados Unidos de América o CDC) y entre estos, 30 tuvieron puntuaciones estándar anómalas en la prueba RCPM; es decir, inferiores al percentil 25. De los 141 niños con concentraciones de plomo mayores de $10 \mu \mathrm{g} / \mathrm{dL}$, 97 tuvieron puntuaciones anómalas en la prueba RCPM. El análisis de regresión mostró una asociación inversa significativa $(r=0,331, P<0,0001)$ entre la concentración de plomo y la puntuación estándar. Cuando se estimaron los CI de los niños mediante la conversión de los puntajes de la RCPM, se estableció una disminución de 2 puntos, aproximadamente, en el CI por cada incremento de $10 \mu \mathrm{g} / \mathrm{dL}$ en la concentración de plomo, desde 10 hasta $>70 \mu \mathrm{g} / \mathrm{dL}$.

Sobre la base de los resultados, los investigadores concluyeron que el rendimiento de los participantes andinos estaba inversamente asociado con la exposición crónica al plomo en los niños con concentraciones de ese elemento por debajo y por encima del nivel de riesgo de $10 \mu \mathrm{g} / \mathrm{dL}$ establecido por los CDC. El descenso en las puntuaciones obtenidas en la prueba apunta a la presencia de una relación entre dosis y respuesta. (Counter SA et al. Neurocognitive impairment in lead-exposed children of Andean lead-glazing workers. J Occup Environ Med. 2005;47:306-12.)

\section{Efectos de la metformina en la composición corporal de personas con factores de riesgo de diabetes tipo 2}

La metformina es un antiglucemiante de administración oral proveniente de las biguanidas, el cual disminuye la producción hepática de glucosa y activa su utilización muscular y su oxidación, así como la de los ácidos grasos en los tejidos periféricos. Entre los efectos farmacológicos más importantes de la metformina cabe mencionar que aumenta la captación de la glucosa en presencia de hiperglucemia o hiperinsulinemia, mejora el funcionamiento de las células $B$, propicia la pérdida de peso y modifica la composición corporal en personas con diabetes tipo 2. Estos cambios se asocian con la reducción de triglicéridos, ácidos grasos y lípidos en todo el cuerpo.

La composición corporal puede medirse con la técnica de impedencia bioeléctrica de resistencia y reactancia a una corriente alterna, que no es invasora sino apropiada para pacientes de cualquier edad, tiene una variación de 1 a $2 \%$ y se correlaciona bien con los métodos de energía atómica. En años recientes se han elaborado índices para determinar la resistencia y la sensibilidad a la insulina según las concentraciones sanguíneas de glucosa e insulina en ayunas. Por otra parte, se recomienda el modelo de homeostasia (homeostatic model assessment-insulin resistance u HOMA-IR) para evaluar la resistencia a la insulina en estudios clínicos y, para evaluar la sensibilidad, el índice cuantitativo (quantitative insulin sensitivity check index o QUICK). Ambas técnicas han sido validadas frente a la pinza euglucémica hiperinsulínica que ha sido el criterio de referencia. En el estudio que presentamos se investigó el efecto de la metformina en la composición corporal así como en la resistencia y sensibilidad a la insulina en pacientes sanos con factores de riesgo de diabetes tipo 2. El estudio se llevó a cabo en el 
Hospital General Regional del Instituto Mexicano de Seguridad Social con la participación inicial de 23 pacientes de ambos sexos. Los factores de riesgo fueron la edad (35 a 40 años), los antecedentes familiares de diabetes tipo 2, no hacer ejercicio y tener un sobrepeso de $10 \%$ o más. Los participantes se asignaron aleatoriamente a recibir $850 \mathrm{mg}$ de metformina o de placebo dos veces al día. Ambos grupos consumían una dieta isocalórica. Cada dos semanas, un experto en nutrición verificaba el cumplimiento del régimen. Antes y después del tratamiento se midieron la composición corporal, la concentración de glucosa sérica en ayunas y la de insulina. Se registraron la estatura, el peso y las circunferencias de cintura y caderas de los pacientes y se calcularon el índice de masa corporal y la relación entre la cintura y las caderas. La resistencia y la sensibilidad a la insulina se midieron con los modelos HOMA-IR y QUICK, respectivamente. Los datos se presentaron como medias y desviaciones estándar y el análisis estadístico para comparar los datos iniciales y finales se basó en la prueba del orden con signo de Wilcoxon, con un intervalo de confianza de $95 \%$, significación de 0,05 y poder de $80 \%$ (paquete estadístico SPSS 9).

Completaron el estudio 12 pacientes del grupo que recibió metformina (8 mujeres y 4 hombres) y 9 del que recibió placebo (4 mujeres y 5 hombres). No hubo cambios estadísticamente significativos entre los grupos en cuanto a ejercicio, peso, relación entre cintura y caderas o lípidos en la sangre. En el grupo que recibió placebo aumentó la concentración final de glucosa sérica que, en un paciente, permitió el diagnóstico de diabetes $(137 \mathrm{mg} / \mathrm{dL})$. Hubo diferencias de base en el contenido corporal de grasa, tejido magro y agua de los dos grupos, que pueden atribuirse al predominio de mujeres. Sin embargo, el tratamiento disminuyó la grasa y aumentó el tejido magro y el contenido de agua, bajando la electroimpedencia generada por los tejidos adiposos. La reducción del porcentaje de grasa corporal no modificó el peso corporal total y se vio compensada por el efecto antigluconeógeno de la metformina y la incorporación muscular de glucógeno. El descenso de la glucosa en ayunas no se acompañó de cambios en los triglicéridos o el colesterol en la sangre. La metformina redujo la resistencia a la insulina por un factor de 7, sin significación estadística ni cambios en la sensibilidad. En conclusión, la metformina modificó la composición corporal disminuyendo la grasa y aumentando el tejido magro y su contenido de agua. Mejoró la resistencia a la glucosa y la redujo en sangre, pero no modificó el peso corporal total, los lípidos en la sangre ni la sensibilidad a la insulina. (Rodríguez-Moctezuma JR et al. Effects of metformin on the body composition in subjects with risk factors for type 2 diabetes. Diabetes Obes Metab. 2005;7:189-92.)

\section{Aculturación y salud de los latinos en los Estados Unidos de América}

Cinco investigadores analizaron lo que se ha publicado sobre el fenómeno de la asimilación de elementos culturales, desde que llegaron los europeos a los Estados Unidos en el siglo XIX hasta la inmigración latinoamericana del siglo XX. La principal deducción fue que la aculturación y sus efectos en la salud de los latinos inmigrantes constituyen un tema tan complejo que todavía no se conoce bien. Por un lado, la salud de los latinos es buena, en general, comparada con la de otros grupos étnicos. En 2001 las tasas de mortalidad de adultos ajustadas por edad fueron $22 \%$ más bajas entre los latinos que entre personas de raza blanca no latinas (PBNL) y $41 \%$ más bajas que entre personas de raza negra no latinas (PNNL). En el mismo año, la mortalidad infantil en la población latina fue similar a la de PBNL y $58 \%$ menor que la de PNNL. Sin embargo, en los tres grupos latinos principales -mexicanos, puertorriqueños y cubanos-, es evidente que la aculturación tiene efectos negativos en ciertos aspectos de la salud y en algunos comportamientos y percepciones, así como en el resultado de los embarazos. En el uso de los servicios preventivos de salud y en la percepción de la propia salud, el efecto es más bien benéfico.

La mayor parte de los estudios se refieren a mexicanos a pesar de que hay importantes variaciones en cuanto a costumbres, estado socioeconómico, escolaridad, fluidez lingüística y estado inmigratorio de los tres subgrupos, incluido el número de generaciones residentes en los Estados Unidos. Los autores destacan una serie de presunciones erróneas en estudios previos: la 'aculturación unidireccional y pérdida final de la cultura original'; el 'control del individuo sobre los procesos de asimilación e integración'; el 'rechazo del familismo' latino y otras, que ocurren individualmente en grados muy distintos o pueden mezclarse. Las medidas de salud y aculturación fallan frente a esa diversidad que se observa no solo en el área temática estudiada, sino en los factores de edad, género, y otros constructos. Muchos estudios sobre los latinos dan resultados incongruentes debido a la adaptación inapropiada de escalas, la diferente comprensión del lenguaje y la inclusión de características sociodemográficas en vez de variables directamente relacionadas con los contextos de la aculturación. Entre subgrupos hay distintos patrones biológicos de predisposición a enfermedades, usos idiomáticos del 